nature of an unsaturated fatty acid, since Meyerhof has shown that the oxidation of linolenic acid is accelerated by the simultaneous oxidation of a sulphydryl group present at the same time. Thus the oxidation of the fatty acid depends on the presence of the reduced glutathione, which in turn is formed by the "hydrogen donators" of the tissues. When oxygen acts as a "hydrogen-acceptor," removing the hydrogen from the reduced glutathione which is thereby oxidised, part of the oxygen becomes activated and available for the oxidation of other substances present in the cell.

\title{
The Royal Observatory, Edinburgh, and Accurate Measurements of Time.
}

THE record of the work done in 1924 at the Royal Observatory, Edinburgh, recorded in the annual report of the Astronomer Royal for Scotland, Prof. R. A. Sampson, is of supreme importance to those interested in the accurate measurement of time.

For some years Edinburgh has taken the lead in this subject, and Prof. Sampson's papers before the Royal Society of Edinburgh and the Royal Astronomical Society on the performance of his clocks have been followed with keen interest in the observatories of the world. His three first-grade clocks are the Riefler No. 258, which, we understand, is one of the best turned out by the famous Munich firm ; Leroy No. I230, which is a duplicate of those at the Paris Observatory used in connexion with the time transmission from the Eiffel Tower; and Shortt No. o, the original Free Pendulum of the Synchronome Co.

By means of an oscillograph and micro-chronograph, which resembles a cinematograph camera, Prof. Sampson's clocks are in effect put under a microscope, that is to say, their performance is observed and automatically recorded each day to an accuracy of one thousandth part of a second, a method which Prof. Sampson says "rarely fails to show when one or other of them has changed rate by as much as oI sec." The daily comparison, he continues, demonstrated a decided superiority in the rate of clock Shortt; indeed the other two were scarcely able to serve as a check upon it. Trerefore, with the view of carrying time work to the limit that the appliances will allow, a second model of the clock (Shortt No. 4) was installed in January in one of the vacant clock cells in the basement. The connexions for temperature control are the same as in the other cells. The original clock of these series (Shortt No. o) which was erected here for trial by Mr. Shortt in I923, and has shown such remarkable going, has been generously presented to the Observatory by the Synchronome Company Limited, in association with whom Mr. Shortt had prepared his model. Briefly, it may be said the only discernible faults in the going of Shortt No. o are a small temperature coefficient showing when an accident disturbs the temperature control of the cell, and a minute leak in the case, which has not been located.

Prof. Sampson's investigations in recent years have focussed attention upon the lack of precision in transit observations, upon which, of course, all time determinations are based. Instrumental errors in the transit circle telescope have always been carefully measured and allowed for. They take a prominent part in the somewhat complex process of smoothing the clock rates. In the race for accuracy, the precision of the clock and of the means of comparison by wireless time signals have outrun the precision of transit observations.

Radio telegraphy has enabled distant observatories to compare their times with great precision, thanks to the rhythmic signals or " time-vernier," and they are found to disagree by amounts which, though they may appear trivial to a layman, are of considerable importance in an exact science such as astronomy. Astronomers have indeed been considerably perturbed at their lack of agreement, and Prof. Sampson had set himself the task of searching for the cause of the error which has been found to occur in time determinations at all observatories.

Prof. Sampson now makes the definite statement that " owing to improvements in the clock and chronograph system in this Observatory, and in receiving apparatus for W.T. signals from other observatories, it has proved possible to bring this investigation to an issue. It has been found that during the whole of I 924 the whole of the large erratic or systematic errors are removed if the level error, determined as usual by the mercury bath, is rejected, and the observations reduced instead by an azimuth error derived from one of the collimators, in combination with the usual observations of polar stars. The implications of this result are of high interest and importance to astronomers and will be pursued."

Referring later to his automatic records of the numerous W.T. signals, Prof. Sampson says, "the comparison of time determined at this observatory with the same determined elsewhere exhibits features of the sub-systematic character that had been noted in previous years, though each observatory had cleared its determinations of all known errors. As remarked above, the source of these discrepancies appears now to be located, at least so far as the observatory is concerned."

\section{World Meteorology and Long-range Forecasting. ${ }^{1}$}

THE possibility of seasonal forecasts in India was first investigated by $\mathrm{H}$. F. Blanford about I876, using only the snowfall in the Himalayas. As the research proceeded, it was found necessary to consider conditions farther and farther away, until in the hands of Sir Gilbert Walker it developed into an investigation of the inter-relationships of weather in all parts of the world. A chart of the average barometric pressure over the globe shows a number of more or less permanent areas of high and low pressure; for example, the Azores high and the Icelandic low. In these areas the variability of pressure reaches a

1 "A Further Study of World-Weather. Applications to Seasonal Frrecasting in India." By Sir G. T. Walker. (Memoirs of the Indian Meteorological Department, vol. 24, Parts 9 and ro.) maximum, and they are accordingly termed " centres of action" ; the pressure changes of intervening places, such as the British Isles, are dominated by those at the centres of action.

Sir Gilbert Walker has realised that these " strategic points " offered the best chances for an attack on the problem of long-range weather forecasting, and has studied in great detail the relations between twenty different centres of action. In "Correlation in Seasonal Variations of Weather," Part 8 (Memoirs of the Indian Meteorological Department), published in I923, he laid down the groundwork of a theory of world weather and outlined the mechanism by which variations in one part of the world are transmitted to other parts of the world a few months later. The

$$
\text { NO. } 29 \text { I } 5 \text {, VOL. I I } 6]
$$

